\title{
Human paraoxonase gene cluster overexpression alleviates angiotensin II-induced cardiac hypertrophy in mice
}

\author{
Jian-Fei Pei, Yun-Fei Yan, Xiaoqiang Tang, Yang Zhang, Shen-Shen Cui, Zhu-Qin Zhang, \\ Hou-Zao Chen ${ }^{*} \&$ De-Pei Liu**
}

State Key Laboratory of Medical Molecular Biology, Department of Biochemistry and Molecular Biology, Institute of Basic Medical Sciences, Chinese Academy of Medical Sciences \& Peking Union Medical College, Beijing 100005, China

Received May 17, 2016; accepted June 21, 2016; published online August 30, 2016

\begin{abstract}
Cardiac hypertrophy is the strongest predictor of the development of heart failure, and anti-hypertrophic treatment holds the key to improving the clinical syndrome and increasing the survival rates for heart failure. The paraoxonase (PON) gene cluster (PC) protects against atherosclerosis and coronary artery diseases. However, the role of PC in the heart is largely unknown. To evaluate the roles of PC in cardiac hypertrophy, transgenic mice carrying the intact human PON1, PON2, and PON3 genes and their flanking sequences were studied. We demonstrated that the PC transgene (PC-Tg) protected mice from cardiac hypertrophy induced by Ang II; these mice had reduced heart weight/body weight ratios, decreased left ventricular wall thicknesses and increased fractional shortening compared with wild-type (WT) control. The same protective tendency was also observed with an Apoe $e^{-1-}$ background. Mechanically, PC-Tg normalized the disequilibrium of matrix metalloproteinases (MMPs)/tissue inhibitors of MMPs (TIMPs) in hypertrophic hearts, which might contribute to the protective role of PC-Tg in cardiac fibrosis and, thus, protect against cardiac remodeling. Taken together, our results identify a novel anti-hypertrophic role for the PON gene cluster, suggesting a possible strategy for the treatment of cardiac hypertrophy through elevating the levels of the PON gene family.
\end{abstract}

cardiac hypertrophy, fibrosis, paraoxonase gene cluster, angiotensin II

Citation: $\quad$ Pei, J.F., Yan, Y.F., Tang, X., Zhang, Y., Cui, S.S., Zhang, Z.Q., Chen, H.Z., and Liu, D.P. (2016). Human paraoxonase gene cluster overexpression alleviates angiotensin II-induced cardiac hypertrophy in mice. Sci China Life Sci 59, 1115-1122. doi: 10.1007/s11427-016-0131-4

\section{INTRODUCTION}

Cardiac hypertrophy, characterized by the enlargement of individual myocytes to augment cardiac pump function and decrease ventricular wall tension, is a compensatory and protective mechanism of cardiomyocytes in response to various physiologic and pathologic stimuli. However, continuous hypertrophic stimulation leads to cardiac dysfunction, during which the ventricle dilates, the myocardial wall thins, and myocardial contractility declines; these phenomena eventually promote the development of heart failure

*Corresponding author (email: chenhouzao@ibms.cams.cn)

**Corresponding author (email: liudp@pumc.edu.cn)
(Frey et al., 2004). Presently, heart failure is acknowledged as one of the leading causes of morbidity and mortality worldwide, and cardiac hypertrophy is the strongest predictor of the development of heart failure (Gardin and Lauer, 2004). Considering that anti-hypertrophic treatment could significantly improve the clinical syndrome and increase survival rates, the discovery of new endogenous negative regulators of cardiac hypertrophy is key to the development of successful therapies for hypertrophic heart disease.

The paraoxonase (PON) gene cluster $(\mathrm{PC})$, which contains three adjacent genes (PON1, PON2, and PON3) (Primo-Parmo et al., 1996), detoxifies oxidized low-density lipoprotein (oxLDL) and homocysteine (Hcy) thiolactone. 
Unlike $P O N 2$ and $P O N 3$, which are expressed ubiquitously in mouse (She et al., 2009), PON1 is expressed primarily in the liver and redistributes to a variety of tissues and organs, including the heart, through circulating high-density lipoprotein (HDL) (Deakin et al., 2011; Marsillach et al., 2008). It has been demonstrated that PC protects against atherosclerosis (She et al., 2009), reduces the incidence of cardiovascular diseases (CVD), and is related to diabetes, metabolic syndrome, and aging via anti-oxidative stress. However, to our knowledge, the role of PC in cardiac hypertrophy has not been reported, and little is known about the reactivity of the PON gene cluster to hypertrophic stimulation.

In the present study using $\mathrm{PC}$-overexpressing transgenic (Tg) (PC-Tg) mice, we showed that PC was a negative regulator in the development of cardiac hypertrophy induced by Ang II. We demonstrated that the mRNA levels of PON2 and $P O N 3$, as well as the protein levels of PON1-3, were upregulated by Ang II infusion for 4 weeks and that PC-Tg repressed cardiac remodeling by modulating the balance of matrix metalloproteinases (MMPs)/tissue inhibitors of MMPs (TIMPs).

\section{RESULTS \\ Changes in the expression of PON1-3 in cardiac hyper- trophy induced by Ang II}

To profile the role of PC in Ang II-induced cardiac hypertrophy, we first assessed the expression patterns of PON1-3 in mouse hearts after Ang II treatment for 4 weeks. We found that the mRNA levels of both PON2 and PON3 were significantly upregulated by Ang II compared with the saline group, whereas PONI's mRNA was absent in both groups of mouse hearts (Figure 1A-C). However, the protein levels of PON1, as well as that of PON2 and PON3, were detected in both the saline and Ang II-treated groups, and they were all markedly upregulated in the hearts from mice infused with Ang II (Figure 1D and E). The immunofluorescence assay showed that PON1 was located mainly on the edge of the myocardium in the control wild-type (WT) mice (Figure 1F). However, after Ang II treatment for 4 weeks, PON1 protein was detectable in the whole myocardium (Figure 1F). Taken together, these results indicate that both the mRNA and protein levels of PON2/3 increased, while PON1 protein redistributed more readily in
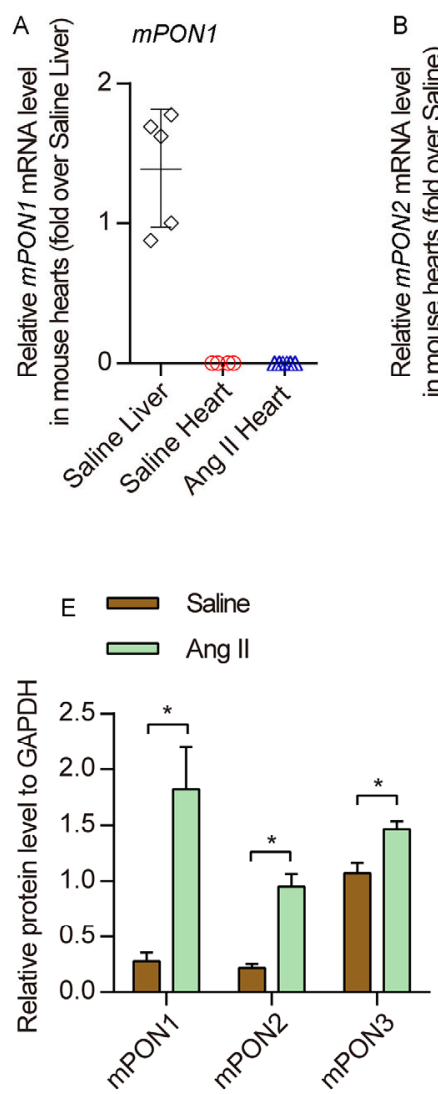
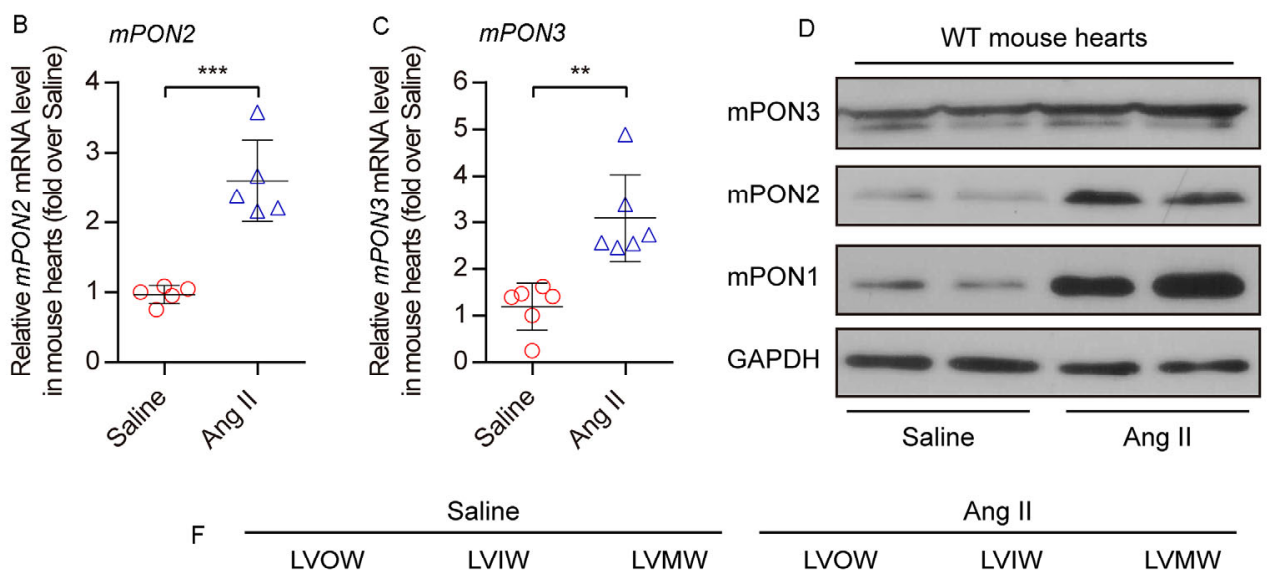

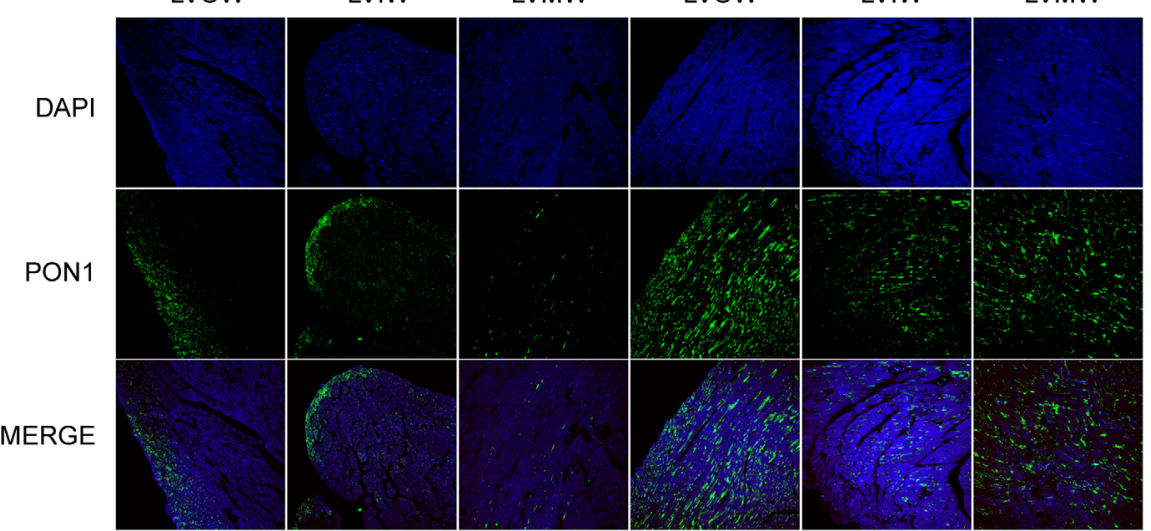

Figure 1 PON gene cluster expression increases in mouse hearts with hypertrophy. A-C, The mRNA of PON1 is not detectable in the mouse hearts (A), whereas the mRNA levels of PON2 (B) and PON3 (C) increase in mouse hearts with hypertrophy induced by angiotensin II (Ang II). The mRNA levels are shown relative to Gapdh. D and E, The protein levels of PON1, PON2 and PON3 increase in the mouse hearts with hypertrophy induced by Ang II. F, PON1 protein is located mainly on the edge of the myocardium in the control WT mice, and after Ang II treatment for 4 weeks, PON1 protein was detectable in the whole myocardium. Eight- to twelve-week-old male wild-type C57LB6 mice were subjected to saline or Ang II infusion for 4 weeks. LVOW, left ventricular outer wall. LVIW, left ventricular inner wall. LVMW, left ventricular mid wall. $n=3-6$ in each group. $*, P<0.05 ; * *, P<0.01 ; * * *, P<0.001$. 
the Ang II-induced hypertrophic hearts, thus indicating the involvement of PC in cardiac hypertrophy.

\section{No noticeable cardiac abnormalities in the PC-Tg mice in the basal state}

To further define the role of PC in cardiac hypertrophy, we used transgenic mice carrying the intact human PON1, $P O N 2$, and $P O N 3$ genes and their flanking sequences (Figure 2A), which were established in our lab (She et al., 2009). Mouse genotyping was performed by PCR and agarose gel electrophoresis (Figure 2B). The tissue-specific expression of the human PON1 (hPON1), hPON2, and $h P O N 3$ transgenes was confirmed (She et al., 2009), and we found that the mRNA of each of $h P O N 1-3$ was detected in the PC-Tg mouse hearts (Figure $2 \mathrm{C}-\mathrm{E}$ ). We did not observe any difference in the heart weight (HW)/body weight (BW) ratios (Figure 2F), cardiac morphology (left ventricular internal diameter at end-diastole, (LVID; d)) (Figure 2G), or cardiac function, including fractional shortening (FS) and ejection fraction (EF) (Figure $2 \mathrm{H}$ and I), between the PC-Tg mice and their non-transgenic littermates. In addition, the levels of hypertrophic fetal gene atrial natriuretic peptide (ANP) between the WT and PC-Tg mice were not significantly different (Figure 2J).

\section{PC-Tg protects mice from Ang II-induced cardiac hy- pertrophy}

To examine the role of the PON gene cluster in the development of cardiac hypertrophy, we subjected the PC-Tg mice and their WT controls to Ang II-induced hypertrophy. No significant difference was observed in blood pressure, heart rate (Table S1 in Supporting Information) and serum lipid profile (data not shown) between the two groups after Ang II treatment. Firstly, we assessed the expression patterns of human PON (hPON) 1-3 in PC-Tg mouse hearts after Ang II treatment for 4 weeks. We noted that only the mRNA and protein levels of hPONI increased significantly by Ang II treatment (Figure $3 \mathrm{~A}$ and D), whereas the expression levels of hPON2 and hPON3 remained unchanged in mouse hearts (Figure 3B-D). Compared with saline-treated littermate controls, Ang II infusion for 4 weeks resulted in massive cardiac hypertrophy in the WT mice, as evidenced by echocardiographic analysis and an increased ratio of $\mathrm{HW}$ to $\mathrm{BW}$ (Figure $3 \mathrm{E}-\mathrm{H}$ ); in contrast, the $\mathrm{PC}-\mathrm{Tg}$ mice showed less significant increases in $\mathrm{HW} / \mathrm{BW}$ ratios (Figure $3 \mathrm{E}-\mathrm{H}$ ). Similarly, the PC-Tg mice were resistant to Ang II-induced thickening of the left ventricular wall (LVW) and decreasing of FS, whereas these were robust in the WT controls (Figure 3I and J). In addition, Ang II treatment significantly
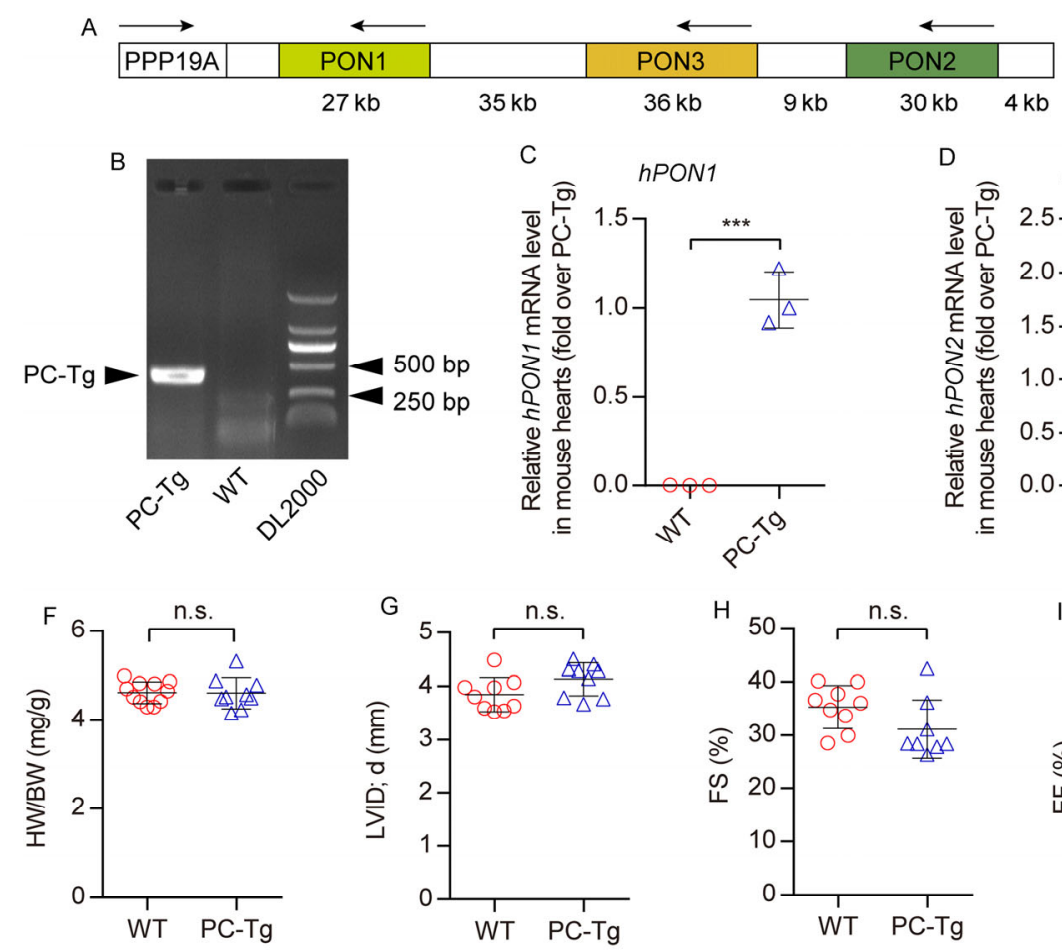
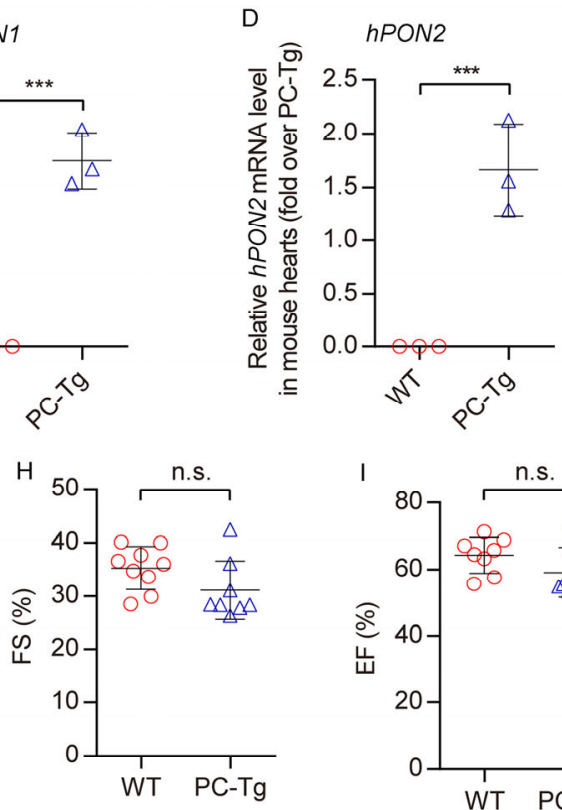
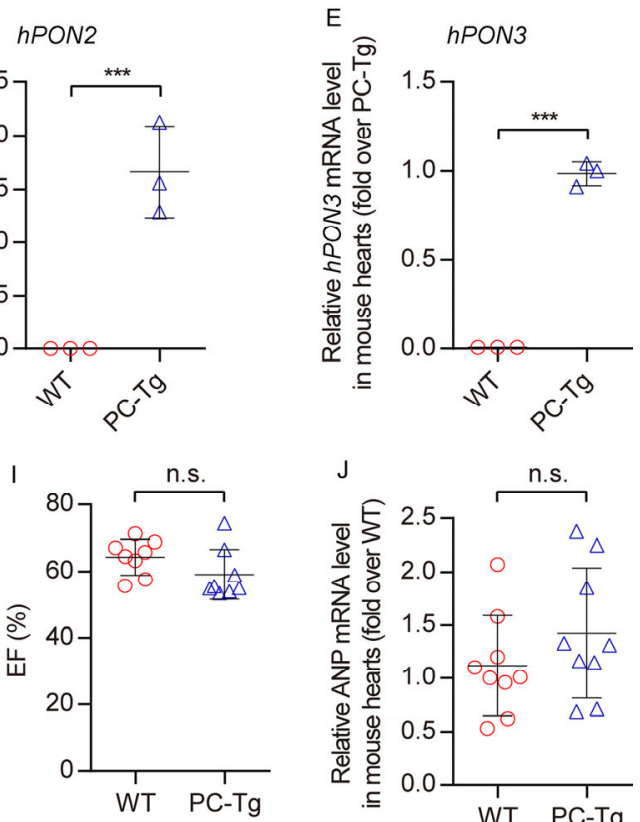

Figure 2 Transgenic (Tg) mice generation. A, A schematic of the Tg construct used to generate the human PC-Tg mouse line. B, Mouse genotyping for PC-Tg was performed by PCR and agarose gel electrophoresis. C-E, Confirmation of the expression of $h P O N 1$ (C), $h P O N 2$ (D), and $h P O N 3$ (E) in the hearts from the PC-Tg mice. The mRNA levels are shown relative to Gapdh. F-J, The PC-Tg mice do not show any difference in heart weight (HW)/body weight (BW) (F), cardiac morphology (left ventricular internal diameter at end-diastole, (LVID; d)) (G), or cardiac function, including fractional shortening (FS) (H), ejection fraction (EF) (I) and mRNA levels of atrial natriuretic peptide (ANP) (J) compared to their WT littermates. $n=3-9$ in each group. n.s., no significance. 

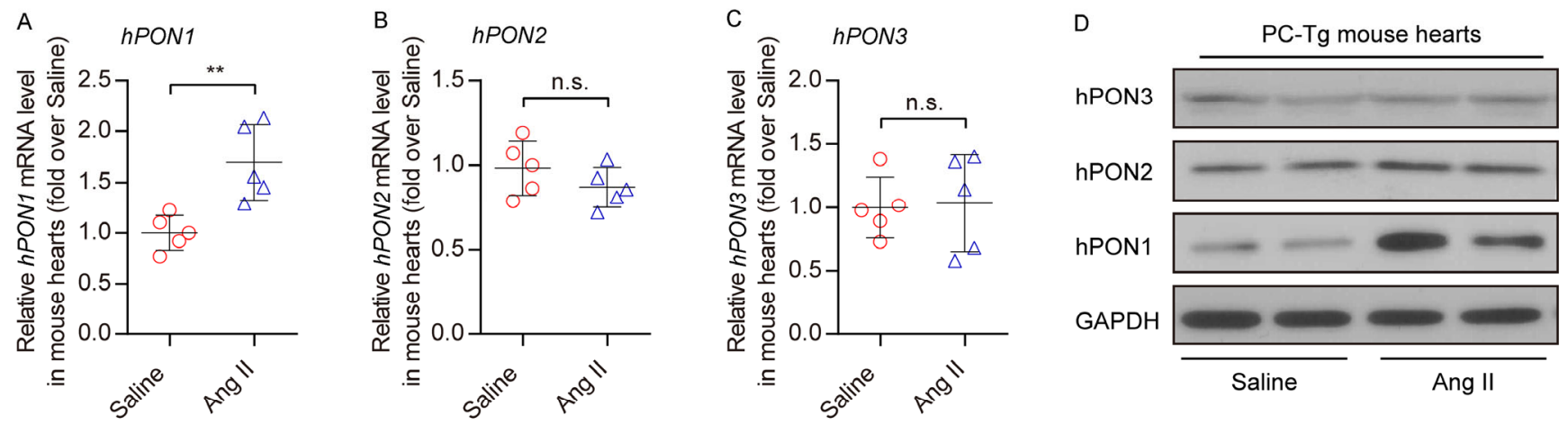

E

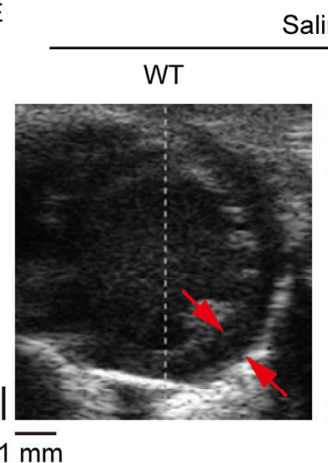

Saline
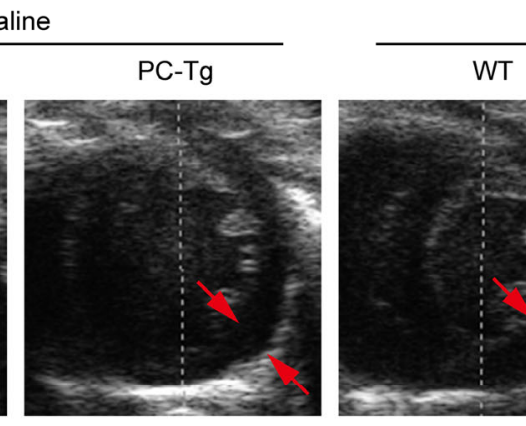

Ang II
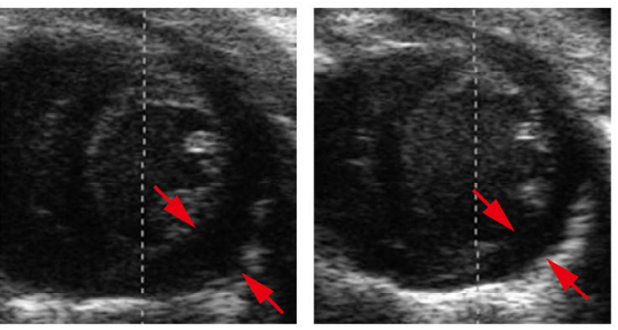

G

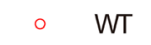

$\triangle \quad$ PC-Tg

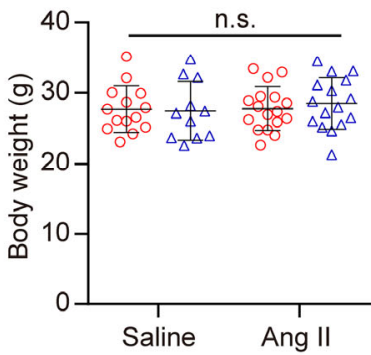

$\begin{array}{lll} & \circ & \text { WT } \\ H & \triangle & \text { PC-Tg }\end{array}$

F
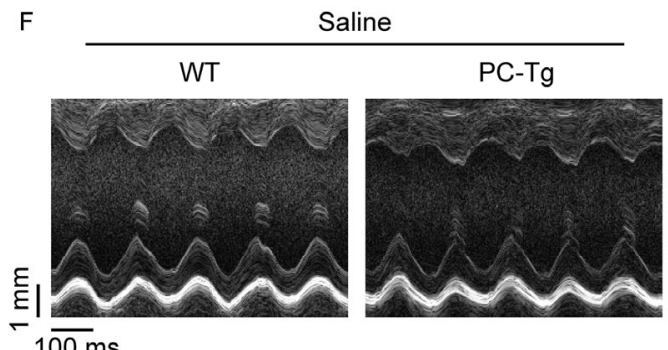

$1 \overline{00 \mathrm{~ms}}$

I $\square$ Saline WT

¿ Saline PC-Tg

¿ Ang II WT

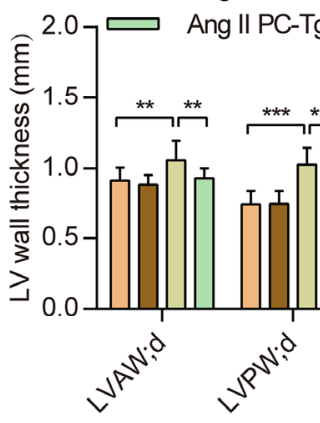

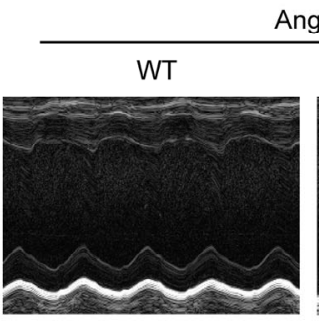

Ang II

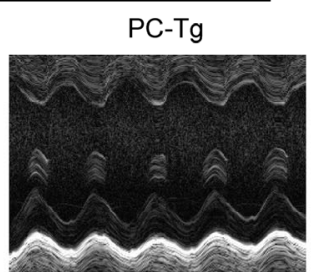

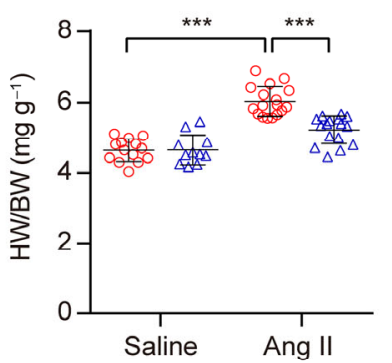

J $\quad \circ \quad W T$

$\triangle \quad \mathrm{PC}-\mathrm{Tg}$

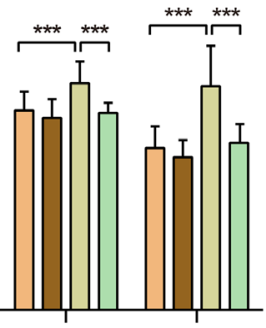

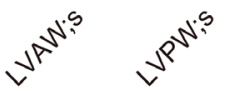

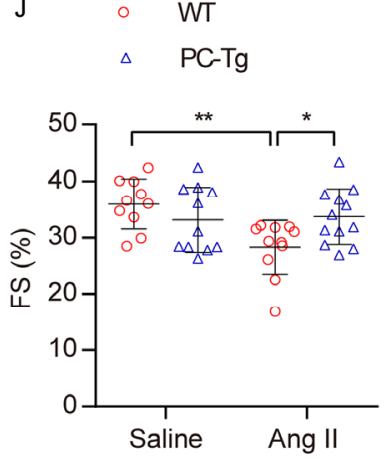

K

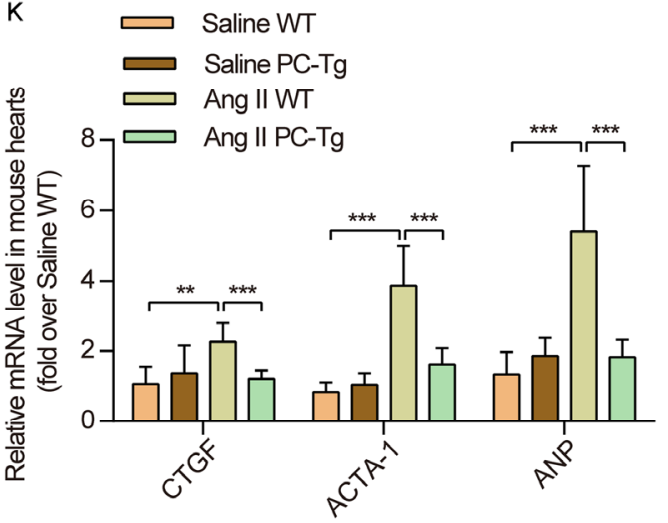

Figure 3 PC-Tg overexpression suppresses Ang II-induced cardiac hypertrophy in WT mice. A-C, The mRNA level of human PON1 ( $h P O N 1$ ) is upregulated in the PC-Tg mouse hearts (A), whereas the mRNA levels of hPON2 (B) and hPON3 (C) remain unchanged in PC-Tg mouse hearts with hypertrophy induced by angiotensin II (Ang II). The mRNA levels are shown relative to Gapdh. D, Representative immunoblot images of hPON1, hPON2 and hPON3 in the saline- or Ang II-induced PC-Tg mouse hearts. E and F, Echocardiographic images showing representative B-mode images (E) and M-mode images (F) from WT and PC-Tg mice treated with saline or Ang II for 4 weeks. G and H, Body weight (BW) (G) and HW/BW ratios (H) of WT and PC-Tg mice treated with saline or Ang II for 4 weeks. I and J, Echocardiographic assessment of the left ventricular wall (LVW) thickness (I) and the FS (J) in saline- and Ang II-treated WT and PC-Tg mice. K, Fetal gene mRNA levels in the hearts of saline- or Ang II-treated WT and PC-Tg mice. WT and PC-Tg mice of 8-12 weeks old were treated with saline or Ang II for 4 weeks. s, systole; d, diastole; PW, posterior wall; AW, anterior wall. $n=5-17$ in each group. n.s., no significance, $*, P<0.05$; **, $P<0.01$; ***, $P<0.001$. 
induced the expression of the fetal genes CTGF, ACTA-1 and ANP in the heart of the WT mice, but no upregulation of these genes was detected in the heart of the PC-Tg mice (Figure 3K).

With the knowledge that the hearts of Apoe $e^{-1-}$ mice exhibit increased ROS and inflammation production (Satoh et al., 2011), we next sought to determine whether PC-Tg protects against the progression of cardiac hypertrophy in the Apoe null backgrounds. PC-Tg Apoe $e^{-1-}$ mice were generated by crossing PC-Tg mice with Apoe $e^{-1-}$ mice (Figure S1 in Supporting Information). Consistent with previous reports (Wang et al., 2005), we found that Ang II infusion for 4 weeks significantly increased cardiac hypertrophy in the Apoe $e^{-1-}$ mice, which was significantly alleviated by the PC-Tg (Figure S2A-D in Supporting Information). These data, taken together, indicate that PC overexpression suppresses the development of cardiac hypertrophy.

\section{Ang II-induced cardiac fibrosis and imbalance of matrix metalloproteinases (MMPs)/tissue inhibitors of MMPs (TIMPs) is retarded in PC-Tg mouse hearts}

During hypertrophy, the myocardial fiber angles and myocyte orientation, which are highly organized and move in a continuous fashion from the endocardium to the epicardium, are replaced by a thickened, poorly organized, myocardial extracellular matrix (ECM) and by hypertrophied myocytes (Spinale, 2007). We then evaluated the cardiomyocyte cross-sectional area and cardiac fibrosis in the hypertrophic hearts using wheat germ agglutinin (WGA) staining and Masson's trichrome staining, respectively, with WT backgrounds. The results showed a decrease in cross-sectional area of the cardiomyocytes and substantially lower levels of fibrosis in the PC-Tg mice than in the WT mice in the Ang II group, while there were no differences between these factors in the saline group (Figure 4A-C).

Given that the disequilibrium of MMPs/TIMPs is the driving force behind ECM homeostasis and cardiac fibrosis during remodeling, we next determined the protein levels of gelatinases (MMP2 and MMP9) (Mann and Spinale, 1998). We found that Ang II significantly increased the protein levels of MMP2/9, as well as TIMP-2, which serves as a receptor for proMMP-2 and promotes its activation by MT1-MMP, in the WT hearts, but these effects were significantly suppressed by PC-Tg (Figure 4D and E). In addition, TIMP1, which binds to the catalytic domain of active MMPs and thereby inhibits MMP activity by preventing the MMPs from accessing their substrates (Spinale, 2007), was upregulated in the hearts of Ang II-treated PC-Tg mice
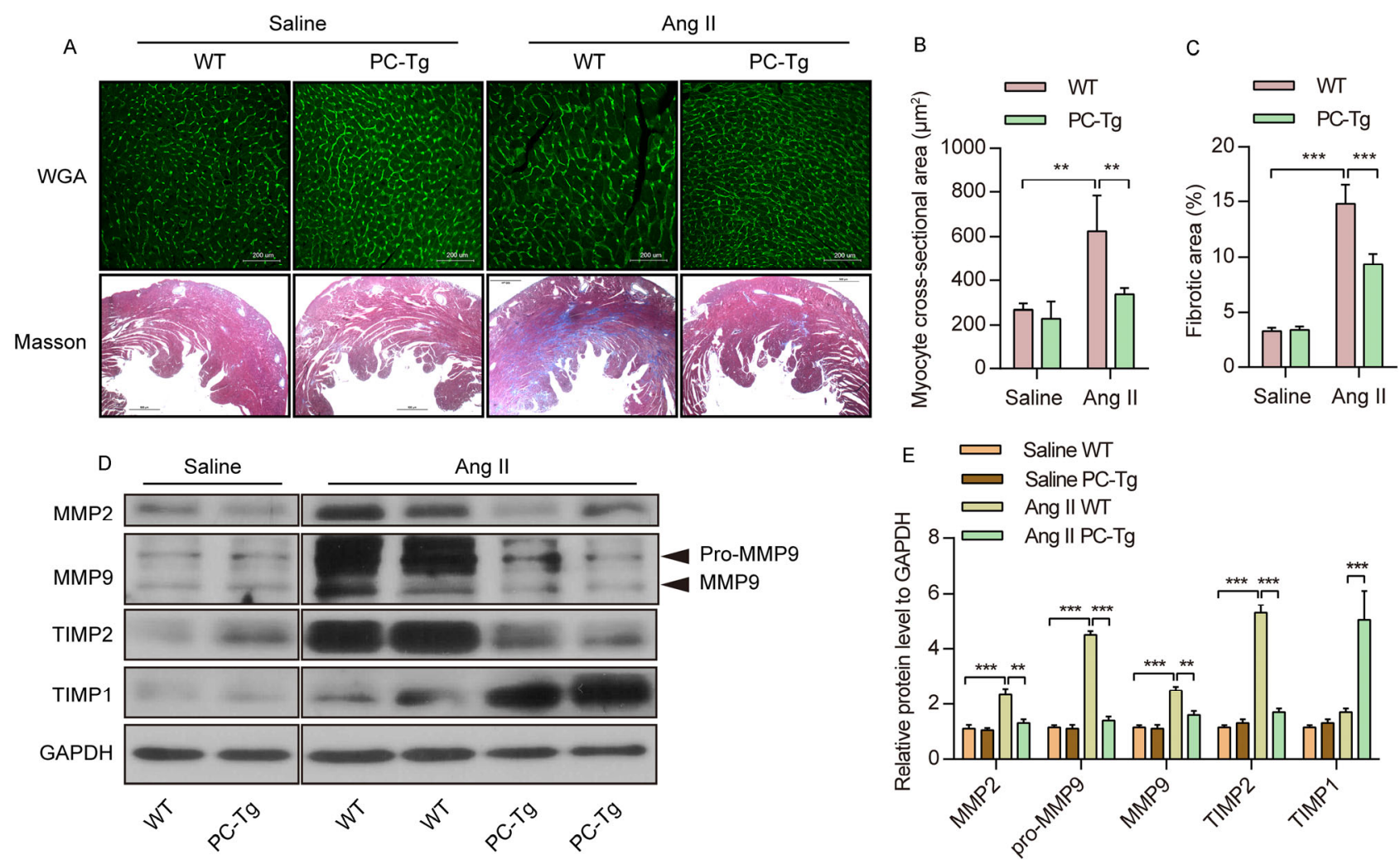

Figure 4 Ang II-induced cardiac fibrosis and imbalance of matrix metalloproteinases (MMPs)/tissue inhibitors of MMPs (TIMPs) is retarded in PC-Tg mouse hearts. A, Histological analysis of the heart sections from the WT and PC-Tg mice following saline or Ang II infusion. The heart cross-sections were stained with WGA (top row; scale bars, $200 \mu \mathrm{m}$ ) and Masson's trichrome staining (bottom row; scale bars, $500 \mu \mathrm{m}$ ). B and C, Quantification of the cardiomyocyte cross-sectional area (B) and the fibrotic area (C) in saline- and Ang II-treated WT and PC-Tg mice. D and E, Representative western blot (D) and quantification (E) of the western blot showing the expression of MMP2, proMMP9, MMP9, TIMP1 and TIMP2 in the hearts from the WT and PC-Tg mice following saline or Ang II infusion. $n=3-8$ in each group. **, $P<0.01$; ***, $P<0.001$. 
compared with those from WT mice (Figure 4D and E).

\section{DISCUSSION}

In the present study, we found that the mRNA and protein levels of PON2/3 were up-regulated, while more of the PON1 protein was redistributed in mouse hypertrophic hearts induced by Ang II for 4 weeks. Furthermore, we have provided the first evidence that PC overexpression alleviates cardiac remodeling and that the mechanisms underlying it might be associated with the suppression of cardiac fibrosis by influencing the imbalance in the MMP/TIMP ratios induced by Ang II.

The PC contains three genes, PON1, PON2, and PON3, all of which share a high sequence identity and a similar protein structure within a given mammalian species (Mackness et al., 2002). However, their tissue and cellular distribution are quite different. In mice, PONI is expressed mainly in the liver, but it can redistribute to a variety of tissues and organs including hearts by associating with HDL and secreting into the serum upon expression in the liver (Deakin et al., 2011; Marsillach et al., 2008). Indeed, we found that PON1 mRNA was absent in the heart but that its level in the liver was high, as previously reported (She et al., 2009). However, PON1 protein was detectable in the WT control hearts and increased significantly in the Ang II-induced hypertrophic hearts. Interestingly, we noted that the location of PON1 migrated from the edge of the myocardium to the internal myocardium after Ang II treatment. Our results imply that the upregulation of PON1 protein in hypertrophic hearts is independent of its mRNA level. Instead, the increase of PON1 protein might be due to the transfer from liver to the heart via HDLs induced by Ang II. These data suggest that PON1 protein is involved in cardiac hypertrophy via its redistribution to the myocardium. Unlike PON1, both PON2 and PON3 are expressed more universally in mice. In spite of the limited research on the transcriptional regulation of the PONs, it was reported that PON2 message and activity were upregulated in mouse livers after these mice were fed a high-fat, high-cholesterol, and cholate-containing diet; in contrast, PON3 expression was not significantly altered compared with their chow-fed counterparts ( $\mathrm{Ng}$ et al., 2005). We are the first to report that both the mRNA and protein levels of PON2 and PON3 in the hearts of mice were upregulated significantly by Ang II treatment for 4 weeks. Taken together, these data indicate that all of the PON gene family members might be involved in cardiac remodeling by increasing their levels in the myocardium, though these increases occur in different manners.

Currently, although there is no general agreement on the natural substrates of PONs, they are recognized as lactonase enzymes. Despite the differences in substrates, all of the PON1/2/3-null mice developed significantly larger atherosclerotic lesions than their WT counterparts when fed a high-fat, high-cholesterol diet (Devarajan et al., 2011; Ng et al., 2006; Shih et al., 1998, 2015). In a previous study, we found that the PC transgene suppressed atherosclerosis development and promoted atherosclerotic plaque stability in an $A$ poe $e^{-1-}$ background (She et al., 2009). Here, we first showed that PONs, which are protective factors for atherosclerosis, are protective against the progression of cardiac hypertrophy induced by Ang II in mice. We demonstrated that PC-Tg suppressed the Ang II-induced increases in $\mathrm{HW} / \mathrm{BW}$ ratios and the thickening of the LVW, as well as a decrease in the FS of the hearts not only from the WT mice, but also from the Apoe $e^{-1-}$ mice. Moreover, the upregulation of fetal gene expression induced by Ang II was significantly suppressed in the hearts of the PC-Tg mice. These findings indicate that the PON gene cluster protects against Ang II-induced cardiac hypertrophy in mice, thus extending our knowledge of the role of PONs from atherosclerosis to cardiac hypertrophy, although additional model such as transaortic constriction (TAC)-induced cardiac hypertrophy is still needed to confirm the protective role of PC-Tg in heart.

Accumulating evidence shows that MMPs and their physiological inhibitors, TIMPs, play a pivotal role in the cardiac remodeling progress (Spinale, 2007). It was recently reported that the transcriptional levels of MMP-2 were upregulated during the compensatory period and that the expression of MMP-9 was increased during the de-compensatory period (Givvimani et al., 2010). The loss or inhibition of MMP-9 suppresses the LV remodeling and dysfunction after acute pressure overload in mice (Heymans et al., 2005a). Among the four TIMPs identified to date, TIMP-2 is unique in that it activates pro-MMP-2 to active MMP-2 and also inhibits the active MMP-2. It was demonstrated that the expression of TIMP-1 and TIMP-2 increased significantly in chronic pressure-overloaded human hearts (Heymans et al., 2005b). When TIMP-1 was knocked out, mice suffered spontaneous LV dilatation, thus indicating the importance of the control of cardiac MMP activity by TIMP-1 (Roten et al., 2000). In the present study, we found that PC-Tg significantly suppressed the Ang II-induced cardiac remodeling, as revealed by the cardiomyocyte crosssectional area and cardiac fibrosis. Consistent with these data, the upregulated levels of MMP2/9 and the MMP2 activator, TIMP2, by Ang II in the hearts of the WT mice were remarkably decreased by PC-Tg, whereas the inhibitor of MMP2/9, TIMP1, was upregulated in the hearts from the PC-Tg mice by Ang II, resulting in a shift of the MMPs/ TIMPs balance to a better status. Taken together, our data support the notion that the PON gene cluster might be an anti-fibrotic factor by repressing the protein levels of MMP2/9 and TIMP2 and upregulating the protein levels of TIMP1.

Interestingly, an association (case-control) study in a United Abrab Emirati population did not find any significant differences in the genotype distribution of the PON1 and PON2 between the LV hypertrophy and non-LV hypertrophy groups (Obineche et al., 2001), whereas another epi- 
demiology data show that the serum arylesterase activity of left ventricular systolic dysfunction patients was higher compared with that in subjects with advanced decompensated heart failure, but lower compared with healthy control subjects. These epidemiology data support the notion that protein levels or the enzymatic activities of PON family members might be associated with cardiac hypertrophy (Tang et al., 2011). Our present work offers the first evidence to shed some light on the protective role of PONs on cardiac hypertrophy in mice. However, further investigations are needed to elucidate the underlying mechanisms. Considering that ROS plays important roles in the initiation and progression of cardiac hypertrophy (Sag et al., 2014), and that PON gene family members are anti-oxidative enzymes (Furlong et al., 2016), it will be important to clarify the anti-ROS function of PC-Tg in the development of cardiac hypertrophy.

In conclusion, we have demonstrated that the PON gene cluster protects against Ang II-induced cardiac hypertrophy possibly through the inhibition of cardiac fibrosis by modulating the balance between MMPs and TIMPs. This discovery suggests a possible strategy for the treatment of cardiac hypertrophy through elevating the levels of PON1-3.

\section{MATERIALS AND METHODS}

\section{Animals}

PC-Tg mice (C57BL/6J background) were constructed, and PC-Tg Apoe ${ }^{-1-}$ mice were generated as described previously (She et al., 2009). All of the animal experiments were performed using 8 - to 12 -week-old male mice. Non-transgenic littermates were used as controls. All of the animal protocols were approved by the Animal Care and Use Committee at the Institute of Basic Medical Sciences, Chinese Academy of Medical Sciences, and Peking Union Medical College (CAMS \& PUMC) (Wang et al., 2016).

\section{Animal models and histological analysis}

Alzet osmotic mini pumps (model 2004; DURECT Corp.) containing $1.44 \mathrm{mg} \mathrm{kg}^{-1} \mathrm{~d}^{-1}$ Ang II (Cat. No. A9525, Sigma-Aldrich, USA) or saline were implanted subcutaneously into the mice for 28 days. The analysis of cardiac hypertrophy, myocyte cross-sectional area, and fibrosis and immunofluorescence measurements were all carried out essentially as routinely done in our laboratory (Luo et al., 2016).

\section{Echocardiography of mice and blood pressure meas- urements}

Echocardiography was performed and analyzed in a blinded manner using a VisualSonics Vevo770 ultrasound biomicroscope (VisualSonics Inc, Canada) with a 15-MHz linear array ultrasound transducer. Mice were anesthetized with saturated tribromoethanol for the duration of the recordings. The left ventricular (LV) was assessed in both the paraster- nal long-axis and short-axis views at a frame rate of $120 \mathrm{~Hz}$. M-mode images were obtained for measuring the left ventricular wall (LVW) thickness, LV end-diastolic diameter (LVEDD) and LV end-systolic diameter (LVESD) at the papillary muscle level. The LV fractional shortening (FS) was calculated as $\mathrm{FS}(\%)=((\mathrm{LVEDD}-\mathrm{LVESD}) / \mathrm{LVEDD}) \times$ 100. The heart rate and systolic blood pressure of the animals were measured using tail-cuff plethysmography (BP-2000 System; Visitech Systems, USA) as described previously (Li et al., 2011).

\section{Quantitative real-time PCR (q-PCR)}

RNA isolation and q-PCR were performed as previously described (Luo et al., 2016), and the q-PCR primers used in this study are listed in the Table S1 in Supporting Information.

\section{Western blot analysis}

Murine cardiac tissues were lysed with RIPA lysis buffer supplied with a mixture of protease inhibitors. The homogenates were sonicated and centrifuged at $4^{\circ} \mathrm{C}$ for $15 \mathrm{~min}$, and the supernatants were used for western blotting. The western blot was performed as described previously (Zhou et al., 2011). The primary antibodies were used for PON1 (Abcam, ab24261), PON2 (Epitomics, S1585), PON3 (Abcam, ab42322), MMP2 (Epitomics, 2008-1), MMP9 (CST, 2270), TIMP1 (Santa, sc-5538), TIMP2 (Abcam, ab1828), GAPDH (Abcam, ab8245).

\section{Statistical analysis}

Data analysis is performed using Graph-Pad Prism Software. All data are presented as the mean \pm SD. The statistical analysis for two groups is performed with an unpaired two-tailed Student's $t$-test. A one-way analysis of variance (ANOVA) followed by a Bonferroni post hoc test is applied for multiple testing. $P$ values less than 0.05 are considered statistically significant.

Compliance and ethics The author(s) declare that they have no conflict of interest.

Acknowledgements This work was supported by the National Natural Science Foundation of China (81422002, 91339201, 31271227, 31571193), the National Science and Technology Support Project (2013YQ0309230502, 2014BAI02B01), and the Beijing Nova Program (XX2013064).

Deakin, S.P., Bioletto, S., Bochaton-Piallat, M.L., and James, R.W. (2011). HDL-associated paraoxonase-1 can redistribute to cell membranes and influence sensitivity to oxidative stress. Free Radic Biol Med 50, 102-109.

Devarajan, A., Bourquard, N., Hama, S., Navab, M., Grijalva, V.R., Morvardi, S., Clarke, C.F., Vergnes, L., Reue, K., Teiber, J.F., and Reddy, S.T. (2011). Paraoxonase 2 deficiency alters mitochondrial function and exacerbates the development of atherosclerosis. Antioxid Redox Signal 14, 341-351.

Frey, N., Katus, H.A., Olson, E.N., and Hill, J.A. (2004). Hypertrophy of 
the heart: a new therapeutic target? Circulation 109, 1580-1589.

Furlong, C.E., Marsillach, J., Jarvik, G.P., and Costa, L.G. (2016). Paraoxonases-1, -2 and -3 : What are their functions? Chem Biol Interact doi: 10.1016/j.cbi.2016.05.036.

Gardin, J.M., and Lauer, M.S. (2004). Left ventricular hypertrophy: the next treatable, silent killer? JAMA 292, 2396-2398.

Givvimani, S., Tyagi, N., Sen, U., Mishra, P.K., Qipshidze, N., Munjal, C., Vacek, J.C., Abe, O.A., and Tyagi, S.C. (2010). MMP-2/TIMP-2/ TIMP-4 versus MMP-9/TIMP-3 in transition from compensatory hypertrophy and angiogenesis to decompensatory heart failure. Arch Physiol Biochem 116, 63-72.

Heymans, S., Lupu, F., Terclavers, S., Vanwetswinkel, B., Herbert, J.M., Baker, A., Collen, D., Carmeliet, P., and Moons, L. (2005a). Loss or inhibition of UPA or MMP-9 attenuates LV remodeling and dysfunction after acute pressure overload in mice. Am J Pathol 166, 15-25.

Heymans, S., Schroen, B., Vermeersch, P., Milting, H., Gao, F., Kassner, A., Gillijns, H., Herijgers, P., Flameng, W., Carmeliet, P., Van de Werf, F., Pinto, Y.M., and Janssens, S. (2005b). Increased cardiac expression of tissue inhibitor of metalloproteinase-1 and tissue inhibitor of metalloproteinase- 2 is related to cardiac fibrosis and dysfunction in the chronic pressure-overloaded human heart. Circulation 112, 1136-1144.

Li, L., Zhang, H.N., Chen, H.Z., Gao, P., Zhu, L.H., Li, H.L., Lv, X., Zhang, Q.J., Zhang, R., Wang, Z., She, Z.G., Zhang, R., Wei, Y.S., Du, G.H., Liu, D.P., and Liang, C.C. (2011). SIRT1 acts as a modulator of neointima formation following vascular injury in mice. Circ Res 108, 1180-1189

Luo, Y.X., Tang, X., An, X.Z., Xie, X.M., Chen, X.F., Zhao, X., Hao, D.L., Chen, H.Z., and Liu, D.P. (2016). Sirt4 accelerates Ang II-induced pathological cardiac hypertrophy by inhibiting manganese superoxide dismutase activity. Eur Heart J doi: 10.1093/eurheartj/ ehw138.

Mackness, B., Durrington, P.N., and Mackness, M.I. (2002). The paraoxonase gene family and coronary heart disease. Curr Opin Lipidol $13,357-362$.

Mann, D.L., and Spinale, F.G. (1998). Activation of matrix metalloproteinases in the failing human heart: breaking the tie that binds. Circulation 98, 1699-1702.

Marsillach, J., Mackness, B., Mackness, M., Riu, F., Beltran, R., Joven, J., and Camps, J. (2008). Immunohistochemical analysis of paraoxonases1,2 , and 3 expression in normal mouse tissues. Free Radic Biol Med $45,146-157$.

Ng, C.J., Bourquard, N., Grijalva, V., Hama, S., Shih, D.M., Navab, M., Fogelman, A.M., Lusis, A.J., Young, S., and Reddy, S.T. (2006). Paraoxonase-2 deficiency aggravates atherosclerosis in mice despite lower apolipoprotein-B-containing lipoproteins: anti-atherogenic role for paraoxonase-2. J Biol Chem 281, 29491-29500.

Ng, C.J., Shih, D.M., Hama, S.Y., Villa, N., Navab, M., and Reddy, S.T. (2005). The paraoxonase gene family and atherosclerosis. Free Radic Biol Med 38, 153-163.

Obineche, E.N., Frossard, P.M., and Bokhari, A.M. (2001). An association study of five genetic loci and left ventricular hypertrophy amongst Gulf
Arabs. Hypertens Res 24, 635-639.

Primo-Parmo, S.L., Sorenson, R.C., Teiber, J., and La Du, B.N. (1996). The human serum paraoxonase/arylesterase gene $(P O N 1)$ is one member of a multigene family. Genomics 33, 498-507.

Roten, L., Nemoto, S., Simsic, J., Coker, M.L., Rao, V., Baicu, S., Defreyte, G., Soloway, P.J., Zile, M.R., and Spinale, F.G. (2000). Effects of gene deletion of the tissue inhibitor of the matrix metalloproteinase-type 1 (TIMP-1) on left ventricular geometry and function in mice. J Mol Cell Cardiol 32, 109-120.

Sag, C.M., Santos, C.X., and Shah, A.M. (2014). Redox regulation of cardiac hypertrophy. J Mol Cell Cardiol 73, 103-111.

Satoh, K., Nigro, P., Zeidan, A., Soe, N.N., Jaffre, F., Oikawa, M., O'Dell, M.R., Cui, Z., Menon, P., Lu, Y., Mohan, A., Yan, C., Blaxall, B.C., and Berk, B.C. (2011). Cyclophilin A promotes cardiac hypertrophy in apolipoprotein E-deficient mice. Arterioscler Thromb Vasc Biol 31, 1116-1123.

She, Z.G., Zheng, W., Wei, Y.S., Chen, H.Z., Wang, A.B., Li, H.L., Liu, G., Zhang, R., Liu, J.J., Stallcup, W.B., Zhou, Z., Liu, D.P., and Liang, C.C. (2009). Human paraoxonase gene cluster transgenic overexpression represses atherogenesis and promotes atherosclerotic plaque stability in ApoE-null mice. Circ Res 104, 1160-1168.

Shih, D.M., Gu, L., Xia, Y.R., Navab, M., Li, W.F., Hama, S., Castellani, L.W., Furlong, C.E., Costa, L.G., Fogelman, A.M., and Lusis, A.J. (1998). Mice lacking serum paraoxonase are susceptible to organophosphate toxicity and atherosclerosis. Nature 394, 284-287.

Shih, D.M., Yu, J.M., Vergnes, L., Dali-Youcef, N., Champion, M.D., Devarajan, A., Zhang, P., Castellani, L.W., Brindley, D.N., Jamey, C., Auwerx, J., Reddy, S.T., Ford, D.A., Reue, K., and Lusis, A.J. (2015). PON3 knockout mice are susceptible to obesity, gallstone formation, and atherosclerosis. FASEB J 29, 1185-1197.

Spinale, F.G. (2007). Myocardial matrix remodeling and the matrix metalloproteinases: influence on cardiac form and function. Physiol Rev 87, 1285-1342.

Tang, W.H., Wu, Y., Mann, S., Pepoy, M., Shrestha, K., Borowski, A.G., and Hazen, S.L. (2011). Diminished antioxidant activity of high-density lipoprotein-associated proteins in systolic heart failure. Circ Heart Fail 4, 59-64.

Wang, F., Chen, H., Yan, Y., Liu, Y., Zhang, S., and Liu, D. (2016). Baicalein protects against the development of angiotensin II-induced abdominal aortic aneurysms by blocking JNK and p38 MAPK signaling. Sci China Life Sci doi: 10.1007/s11427-015-0277-8.

Wang, Y.X., da Cunha, V., Martin-McNulty, B., Vincelette, J., Li, W., Choy, D.F., Halks-Miller, M., Mahmoudi, M., Schroeder, M., Johns, A., Light, D.R., and Dole, W.P. (2005). Inhibition of Rho-kinase by fasudil attenuated angiotensin II-induced cardiac hypertrophy in apolipoprotein E deficient mice. Eur J Pharmacol 512, 215-222.

Zhou, S., Chen, H.Z., Wan, Y.Z., Zhang, Q.J., Wei, Y.S., Huang, S., Liu, J.J., Lu, Y.B., Zhang, Z.Q., and Yang, R.F. (2011). Repression of P66Shc expression by SIRT1 contributes to the prevention of hyperglycemia-induced endothelial dysfunction. Circ Res 109, 639-648.

Open Access This article is distributed under the terms of the Creative Commons Attribution License which permits any use, distribution, and reproduction in any medium, provided the original author(s) and source are credited.

\section{SUPPORTING INFORMATION}

Table S1 Blood pressure and heart rate of WT and PC-Tg mice following saline or Ang II infusion for 4 weeks.

Table S2 The primers for real-time PCR

Figure S1 Mouse genotyping for Apoe $e^{-1-}$ was performed by PCR and agarose gel electrophoresis.

The supporting information is available online at life.scichina.com and link.springer.com. The supporting materials are published as submitted, without typesetting or editing. The responsibility for scientific accuracy and content remains entirely with the authors. 